\title{
A Search for Pulsation in Very Low-mass Stars and Brown Dwarfs
}

\author{
Ann Marie Cody \\ California Institute of Technology, MC 105-24, Pasadena, CA 91125
}

\begin{abstract}
Brown dwarfs and very low-mass stars constitute a crucial link between the intertwined processes of star formation and planet formation. To date, however, observational methods to uncover their formation mechanism or determine important properties such as mass and age have been lacking. Pulsation powered by deuterium burning in brown dwarfs and very low-mass stars is a newly suggested phenomenon that offers unprecedented opportunities to probe the interiors and evolution of these objects. We report on a photometric campaign to search for low-amplitude pulsations among young star-cluster members using a number of telescopes.
\end{abstract}

Keywords: stars: low-mass, brown dwarfs - galaxies: star clusters - stars: oscillations - techniques: photometric

PACS: $97.10 . \mathrm{Sj}, 97.20 . \mathrm{Vs}, 95.75 . \mathrm{Wx}$

\section{INTRODUCTION}

The study of young star clusters ( $\lesssim 10^{8} \mathrm{Myr}$ ), and in particular, characterization of their low-mass stellar and substellar members, has received increasing attention with the advent of near-infrared photometric surveys and focused spectroscopic techniques. These regions are an important arena for testing scenarios for the formation and subsequent evolution of stars, brown dwarfs, and planets. Not only are they attractive for the rich variety of ongoing phenomena hinted at by X-ray activity, photometric variability, and circumstellar disk signatures, but they are also appealing from a statistical standpoint: the mass-dependent properties of young-cluster members can be assessed under the assumption that they were born at roughly the same time and were subject to similar environmental processes. Substantial populations of very low-mass stars (VLMSs) and brown dwarfs (BDs) recently have been identified in open clusters and star-forming regions (e.g., Comerón et al. 2004; Luhman 2004a). With masses intermediate to those of stars and planets, brown dwarfs provide a crucial link in our understanding of star and planet formation.

Theoretical models (Burrows et al. 1997, Baraffe et al. 2003) are moderately successful in reproducing observed photospheric properties of BDs and VLMSs that are derived from standard spectroscopic and imaging techniques. By incorporating the physics of convection, degeneracy, and atmospheres, these authors generate isochrones that exhibit temperatures and luminosities consistent with those seen in young-cluster objects. While such models can be used to infer masses and ages for young, low-mass objects, they have not been extensively tested by independent measurements.

To date, there are only two accurate dynamical mass measurements for BDs, thanks to the discovery of a young $(\sim 1-15 \mathrm{Myr})$, low-mass $\left(\sim 0.03 \mathrm{M}_{\odot}\right)$ eclipsing binary system 
in the Orion nebula star-forming region (Stassun et al. 2006). The results imply intriguing discrepancies between the observed and predicted BD parameters, in particular the radii and temperatures. The identification of further eclipsing systems will illuminate this problem, but unfortunately it cannot provide a direct assessment of the interior conditions such as convection and equation of state that are critical to theoretical models. Stellar seismology, on the other hand, is a tool that has shown great promise in filling this gap. As demonstrated for more-massive stars, the identification of stellar oscillation modes enables a detailed comparison of temperature and density profiles, as well as convective properties, with theory.

We suggest that the detection and characterization of pulsations in BDs and VLMSs offers a superb orthogonal technique for investigating the interiors of these objects and anchoring the low-mass end of the models upon which all studies of the IMF and of star formation histories depend. Here we present a campaign to search for low-amplitude oscillations in low-mass objects across a range of ages and star clusters.

\section{OBSERVATIONAL CAMPAIGN}

\subsection{Background}

Seismology has achieved great success as a tool in the study of the interiors and evolution of many classes of stars, from $\delta$-Scuti stars to pulsating white dwarfs. While it has yet to be put to use in brown dwarfs, several recent developments motivate the exciting new prospect of searching for pulsations in these objects. An analysis by Palla $\&$ Baraffe (2005) has hypothesized that young BDs possess unstable, growing oscillation modes which are fed in part by the steep temperature dependence of deuterium burning. They predict an instability strip on the H-R diagram that extends from low-mass brown dwarfs ( $\mathrm{M} \sim 0.02 \mathrm{M}_{\odot}$ ) oscillating with periods of approximately one hour to very lowmass stars $\left(\mathrm{M} \sim 0.1 \mathrm{M}_{\odot}\right.$ ) with $\sim 4$-hour pulsations. Intriguingly, the temperatures and luminosities of quite a few of the currently known BDs and VLMSs in young star clusters fall within the unstable region, to within the precision of the observations; an example of data from a number of clusters is shown in Figure 1. This suggests the exciting new possibility of conducting a statistically significant search for pulsation.

While the phenomenon has yet to be definitively confirmed, several studies in the literature report variability in BDs suggestive of pulsation, even though the authors of record did not interpret it as such. Substellar members of the $\sigma$-Orionis cluster were observed by Bailer-Jones \& Mundt (2001), Zapatero-Osorio et al. (2003), and Caballero et al. (2004), and variability appears on the appropriate time scale of several hours. Nevertheless, the origin of periodicity in brown-dwarf lightcurves has most often been attributed to rotation and not thoroughly investigated on short time scales. As such, pulsation in brown dwarfs is an unconfirmed phenomenon, and additional high-precision photometry on young, low-mass objects will test the promising theoretical results and provide many more insights. 


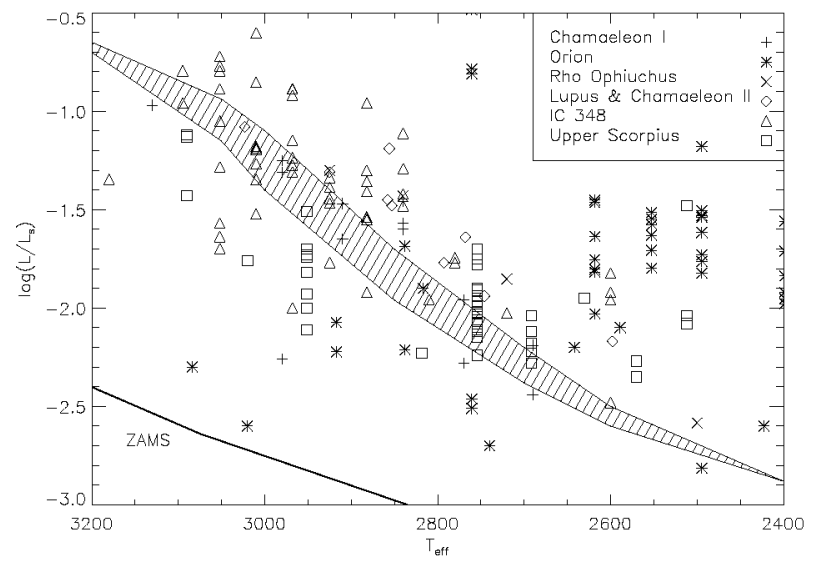

FIGURE 1. The instability strip for unstable brown dwarfs and very low mass stars from PB05 is shown, along with a number of confirmed low-mass cluster members identified by Allers et al. (2006), Luhman (1999), Luhman \& Rieke (1999), Comerón et al. (2004), Slesnick et al. (2004), Cushing et al. (2000). The zero-age main sequence is shown for comparison. With typical errors $100 \mathrm{~K}$ in temperature, and $0.1 \mathrm{dex}$ in $\log$ luminosity, many of the data points lie on the strip, suggesting that they might exhibit pulsation.

\subsection{Feasibility}

To augment the existing data, we have designed first dedicated search (to our knowledge) for pulsation-induced variability in young-cluster brown dwarfs and low-mass stars. The lack of known large-amplitude brown-dwarf pulsators suggests that shortperiod photometric variability in these objects may occur at a level below 0.01-0.02 magnitudes. Therefore, searches for pulsation will likely need to achieve high photometric precision over a substantial number of data points. To determine the feasibility and limits of detecting low-amplitude pulsations with various ground and space-based telescopes, we have performed simulations of periodic light curves with added Gaussian noise. In order to detect a pulsation mode at greater than $99 \%$ confidence, a signal-tonoise ratio of at least 4.0 is required in the Fourier transform of time series data (e.g., Breger 1993). We have generated artificial light curves with periods of 1-4 hours (as expected for this type of pulsation), and added Gaussian noise at a level typical of either ground-based CCDs $(\sim 0.01$ magnitudes) or space-based instruments $(\sim 0.005$ magnitudes). Adopting typical observing cadences and total times, we obtain corresponding sets of artificial data and Fourier spectra, from which the known pulsation signal can be extracted and its significance evaluated. We illustrate example simulations of data expectations from various telescopes in Figures 2 and 3. 


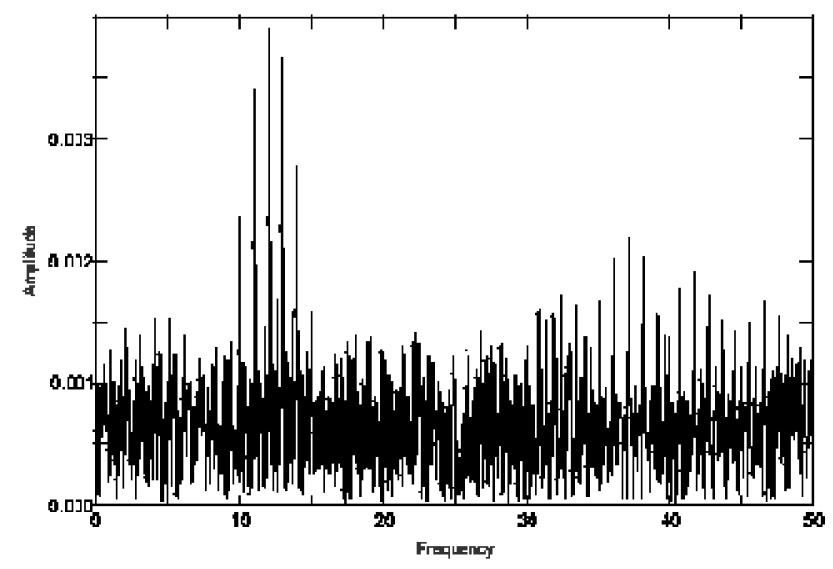

FIGURE 2. We simulate the Fourier periodogram of a lightcurve with a 2-hour period (frequency $=12$ cycles/day) and added Gaussian noise. This simulation assumes 14 nights of ground-based data with 6 hours each, taken at a cadence of 7 minutes per exposure and typical estimated photometric precision of 0.01 magnitudes. The peak at $12 \mathrm{cycles} / \mathrm{day}$ is visible at a signal-to-noise ratio of 4.3 ; the peaks surrounding it are aliases due to daily gaps in the data and can be easily removed, as shown by our simulations.

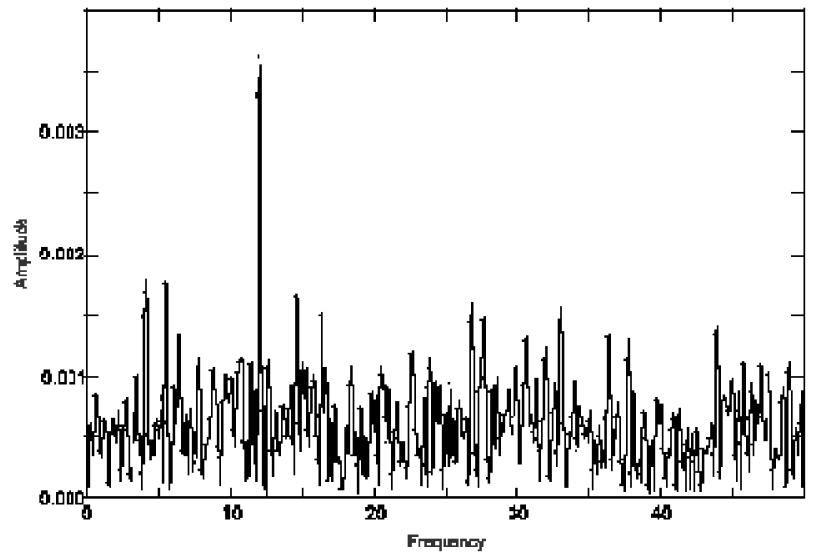

FIGURE 3. We simulate the fourier periodogram of A periodogram resulting from data taken by a ground-based global telescope network with continuous observing at 0.01 magnitude precision for 4 days. The lightcurve parameters are the same as in Figure 2. The expected peak at 12 cycles/day appears at $\mathrm{S} / \mathrm{N}=4.6$. 
The signal-to-noise ratio in the Fourier domain is given roughly by

$$
\frac{S}{N}=\left(\frac{A}{2 \sigma}\right) \sqrt{N},
$$

where $A$ is the pulsation amplitude, $\sigma$ is the RMS error in a typical magnitude measurement, and $N$ is the total number of data points. $N$ can also be specified by $L \times f / c$, where $L$ is the number of nights of data, $f$ is the fraction of each day available to observations, and $c$ is the photometric cadence in data points. While Equation 1 yields similar $S / N$ for large $L$ and small $f$ (many nights of observing with sparse data collection) as it does for smaller $L$ and larger $f$ (a few nights with excellent coverage), the latter is more favourable since our ability to resolve signals in frequency space depends on the longest uninterrupted time series. Signal width is proportional to $1 / f$, and the lower the frequency resolution, the higher the chance that multiple signals will be blended together.

To estimate what sorts of pulsation amplitudes will be detectable via ground-based photometry, we take the example of a 14-night observing run with 8 hours of data per night and 6-minute photometric cadence. This yields 112 hours of photometry and a total number of data points $N=1120$. Previous ground-based observing campaigns (e.g., Hartman et al. 2005; Everett \& Howell 2001; Gilliland 1993) have demonstrated that submilli-mag photometric precision is possible on stars as $\operatorname{dim}$ as $R=16.3$. Atmospheric scintillation prevent ground-based photometric from achieving better than fractional milli-mag precision, while second-order extinction effects raise this limit further in the near-infrared bands. While our brown-dwarf targets are generally quite dim $(I=15$ to 21 ), we nevertheless believe that precisions of $\sigma=0.005 \mathrm{mag}$ will be readily achievable from the ground by effectively removing cosmic rays. Using $N=1120$ and $\sigma=0.005$ in Equation 1, and setting the signal-to-noise ratio to be at least 4.0, we find that we can potentially detect pulsations with amplitudes as low as $0.001 \mathrm{mag}$.

We note that while spectroscopic monitoring is often the method of choice for observing pulsation in other classes of objects (there is much less atmospheric noise at high frequencies and the signals are within detectable limits), the necessary radial velocity precision is probably too high to carry out an extensive and efficient campaign on objects as dim as brown dwarfs. Although it is not entirely straightforward to compute radialvelocity amplitudes from photometric variations, Kjeldsen \& Bedding (1995) provide a method to relate the two, under the assumption that temperature variations dominate radius changes in the pulsation; their results are consistent with observations of many pulsators, including the $\beta$-Cephei stars, RR Lyraes, and $\delta$-Scuti stars. For $I$-band photometric variations of 0.005 magnitudes on an object with $T_{\text {eff }} \sim 2400-3100 \mathrm{~K}$, their prescription yields expected radial velocity amplitudes of approximately $60-100 \mathrm{~m} \mathrm{~s}^{-1}$. In comparison to the $20-30 \mathrm{~cm} \mathrm{~s}^{-1}$ signals detectable in nearby solar-like stars, these values are relatively large. But at the faint magnitudes of brown dwarfs, few current near-IR instruments are able to obtain this level of accuracy in exposures short enough to acquire multiple data points per pulsation period. 


\subsection{Photometric Monitoring Program}

To amass a statistically-significant sample of observations of BDs and VLMSs, our photometric monitoring program will involve a number of small to medium-sized telescopes. Time on the Palomar 60-inch and CTIO 1.0-m telescopes has already been acquired for late 2007. A data reduction pipeline using the VAPHOT aperture photometry package (Deeg et al. 1998) along with cosmic ray removal has been streamlined and regularly produces photometric precisions of under 0.01 magnitudes for $\operatorname{dim}(I=17-20)$ targets. Data will be taken at cadences of 2-10 minutes, since periodicities as short as one hour could be present. Initially, we will observe primarily in the $I$ band, since substellar objects are brightest in the near-infrared. However, we will also obtain a subset $R$ band observations, since it has been suggested that the abundant $\mathrm{TiO}$ absorption features present in late-type stars may make variability amplitudes larger at shorter wavelengths (Percy et al. 2001; Matai 2007).

Since pulsational instability is predicted to occur only within a small region of the H-R diagram, our chances of identifying the phenomenon increase greatly with sample size. Thus, we aim to survey a significant fraction of the previously identified very low-mass objects in young star-forming regions, and obtain a statistically complete census of lowamplitude variability in this population. The most promising search regions, including Taurus, Chamaeleon I, Upper Scorpius, IC 348, $\sigma$ Orionis, $\lambda$ Orionis, and Orion OB1b, encompass estimated ages of 1 to $10 \mathrm{Myr}$. This range will enable us to test the effect of not only mass, but also evolution, on our results, ultimately enabling a comparison of observed trends with pulsation theory predictions. A compilation of these regions and their more than 250 very low-mass members is given in Table 1 . The $0-10 \mathrm{Myr}$ range of these clusters will enable us to test the effect of not only mass, but also age, on our results, ultimately enabling a comparison of observed trends with pulsation theory predictions.

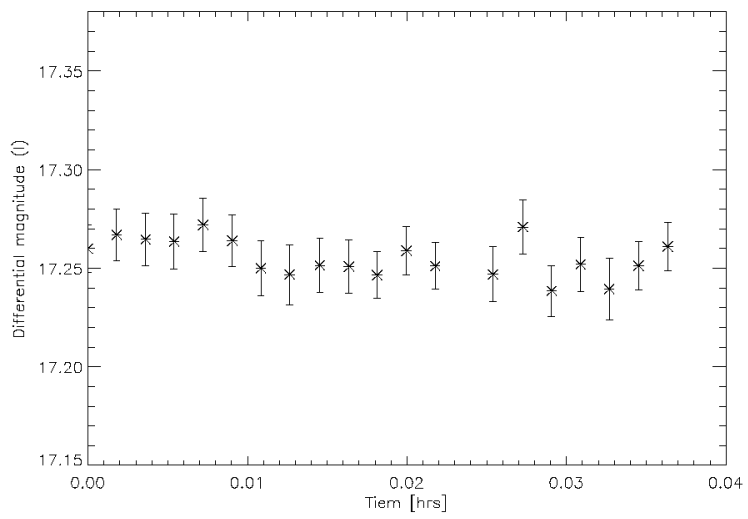

FIGURE 4. A short sample light from an observation of a young brown dwarf shows a photometric precision of 0.008 magnitudes. 
TABLE 1. The sample of spectroscopically-confirmed and likely-candidate brown dwarfs and very low-mass stars (0.02$0.1 \mathrm{M}_{\odot}$ ) in nearby star-forming clusters. References are as follows: (1) Luhman (2004b), Luhman et al. (2006), Martín et al. (2001), Guieu et al. (2005), Briceño et al. (2002), Luhman et al. (2003a), Briceño et al. (1998); (2) Luhman et al. (2004a), Comerón et al. (2004); (3) Martín et al. (2004), Slesnick et al. (2006), Ardila et al. (2000), Preibisch et al. (2002); (4) Luhman (1999), Luhman et al. (2003b), Luhman et al. (2005); (5) Cushing et al. (2000), Wilking et al. (1999); (6) Barrado y Naváscues (2003), Béjar et al. (2004); (7) Barrado y Naváscues et al. (2004); (8) Scholz \& Eislöffel (2005); (9) Comerón et al. (2003), Allen et al. (2007).

\begin{tabular}{cccc}
\hline Cluster & Age & Number of BDs & References \\
\hline Taurus & $1-3 \mathrm{Myr}$ & 55 & 1 \\
Chamaeleon I & $1-3 \mathrm{Myr}$ & 14 & 2 \\
Upper Scorpius & $5 \mathrm{Myr}$ & 91 & 3 \\
IC 348 & $2-3 \mathrm{Myr}$ & 22 & 4 \\
$\rho$ Ophiuchus* & $0-2 \mathrm{Myr}$ & 27 & 5 \\
$\sigma$ Orionis & $5 \mathrm{Myr}$ & 37 & 6 \\
$\lambda$ Orionis & $4-6 \mathrm{Myr}$ & 20 & 7 \\
Orionis OB1b* & $8-10 \mathrm{Myr}$ & 11 & 8 \\
Lupus & $1 \mathrm{Myr}$ & 10 & 9 \\
\hline
\end{tabular}

* clusters lack spectroscopically confirmed brown dwarfs

\subsection{Preliminary Observations}

We have used the Palomar 60-inch telescope to perform initial tests of the observing set-up and data reduction on several BDs with spectral types M6-M8 in the Upper Scorpius association. Preliminary results indicate that any short-timescale periodic variability in these objects indeed occurs at very-low amplitude. We have confirmed that photometric precision of less than 0.01 magnitudes is readily achievable with an ensemble of five or more reference stars. An example of the $I$-band photometric is presented in Figure 4.

\section{SUMMARY}

In summary, we have developed a program to both search for and classify pulsations in brown dwarfs and very low-mass stars, with the end goal of integrating the observations into a more comprehensive theory. If detected, pulsations in BDs and VLMSs will not only open up a new instability-strip on the H-R diagram, but also represent the first confirmation of the $\varepsilon$ mechanism. Ultimately, we hope that this work will provide a way to correlate observational data with the fundamental properties of these objects. 


\section{ACKNOWLEDGMENTS}

The author would like to thank P.E.O. International for a generous research and travel award.

\section{REFERENCES}

Allen P. R., Luhman K. L., Myers P. C., Megeath S. T., Allen L. E., Hartmann L., Fazio G. G., 2007, ApJ, 655,1095

Allers K. N., Kessler-Silacci J. E., Cieza L. A., Jaffe D. T., 2006, ApJ, 644, 364

Ardila D., Martín E., Basri G., 2000, AJ, 120, 479

Bailer-Jones C. A. L., Mundt R., 2001, A\&A, 367, 218

Barrado y Navascués D., Béjar V. J. S., Mundt R., Martín E. L., Rebolo R., Zapatero Osorio M. R., Bailer-Jones C. A. L., 2003, A\&A, 404, 171

Barrado y Navascués D., Stauffer J. R., Bouvier J., Jayawardhana R., 2004, astro-ph/041 1438

Baraffe I., Chabrier G., Barman T. S., Allard F., Hauschildt P. H., 2003, A\&A, 402, 701

Béjar V. J. S., Zapatero Osorio M. R., Rebolo R., 2004, AN, 325, 705

Breger M., 1993, IAU Coll., eds. C.J. Buttler \& I. Elliott, 36, 106

Briceño C., Hartmann L., Stauffer J., Martín E., 1998, AJ, 115, 2074

Briceño C., Luhman K. L., Hartmann L., Stauffer J. R., Kirkpatrick J. D., 2002, ApJ, 580,317

Burrows A., Hubbard W. B., Lunine J. I., Marley M., Guillot T., Saumon D., Freedman R. S., 1997, ASP Conference Series, Vol. 119, ed. D. Soderblom, 9

Caballero J. A., Béjar V. J. S., Rebolo R., Zapatero Osorio M. R., 2004, A\&A, 424, 857

Comerón F., Fernández M., Baraffe I., Neuhäuser R., Kaas A. A., 2003, A\&A, 406, 1001

Comerón F., Reipurth B., Henry A., Fernández M., 2004, A\&A, 417, 583

Cushing M. C., Tokunaga A. T., Kobayashi N., 2000, AJ, 119, 3019

Deeg H. J., Doyle L. R., Kozhevnikov V. P., et al., 1998, A\&A, 338, 479

Everett M. E., Howell S. B., 2001, PASP, 113, 1428

Gilliland R. L., Brown T. M., Kjeldsen H., et al., 1993, AJ, 106, 2441

Guieu S., Monin J.-L., Dougados C., Magnier E., Martín E. L., 2005, AN, 326, 1068

Hartman J. D., Stanek K. Z., Gaudi B. S., Holman M. J., McLeod B. A., 2005, AJ, 130, 2241

Kjeldsen H., Bedding T. R., 1995, A\&A, 293, 87

Luhman K.L., 1999, ApJ, 525, 466

Luhman K.L., 2004a, ApJ, 602, 816

Luhman K.L., 2004b, ApJ, 617, 1216

Luhman K. L., Briceño C., Stauffer J. R., Hartmann Lee, Barrado y Navascués D., Caldwell N., 2003a, ApJ, 590, 348

Luhman K. L., Lada E. A., Muench A. A., Elston R. J., 2005, ApJ, 618, 810

Luhman K.L., Rieke G.H., 1999, ApJ, 525, 440

Luhman K. L., Stauffer J. R., Muench A. A., Rieke G. H., Lada E. A., Bouvier J., Lada C. J., 2003b, ApJ, 593,1093

Luhman K. L., Wilson J. C., Brandner W., et al., 2006, ApJ, 649, 894

Martín E. L., Delfosse X., Guieu S., 2004, AJ, 127, 449

Martín E. L., Dougados C., Magnier E., Ménard F., Magazzù, A., Cuillandre J.-C., Delfosse X., 2001, ApJ, 561, L195

Matai M., 2007, ApJ, 113, 1633

Palla F., Baraffe I., 2005, A\&A, 432, 57

Percy J. R., Wilson J. B., Henry G. W., 2001, PASP, 113, 983

Preibisch T., Brown A. G. A., Bridges T., Guenther E., Zinnecker H., 2002, AJ, 124, 404

Scholz A., Eislöffel J., 2005, A\&A, 429, 1007

Slesnick C. L., Carpenter J. M., Hillenbrand L. A., 2006, AJ, 131, 3016

Slesnick C. L., Hillenbrand L. A., Carpenter J. M., 2004, ApJ, 610, 1045

Stassun K. G., Mathieu R. D., Valenti J. A., 2006, Nat, 440, 311

Wilking B. A., Greene T. P., Meyer M. R., 1999, AJ, 117, 469

Zapatero Osorio M. R., Caballero J. A., Béjar V. J. S., Rebolo R., 2003, A\&A, 408, 663 